\title{
Distribución actual y potencial de la guacamaya verde en Michoacán, México
}

\section{Present and potential distribution of the military macaw in Michoacán, Mexico}

\author{
Tiberio C. Monterrubio-Rico ${ }^{\bowtie}$, Miguel Ángel De Labra-Hernández, Juan Manuel Ortega-Rodríguez, Ramón \\ Cancino-Murillo y José Fernando Villaseñor-Gómez

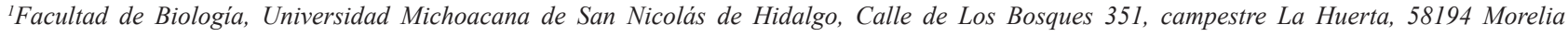 \\ Michoacán, México. \\ 凶tiberio@umich.mx
}

Resumen. La guacamaya verde (Ara militaris) es una especie considerada en peligro de extinción en México y ha desaparecido en estados del Pacífico sur. Sin embargo, no existen estudios actualizados sobre su distribución en los estados donde todavía se puede encontrar. El objetivo principal de este estudio fue conocer su distribución en Michoacán. Se evaluaron 215 localidades de 3 regiones fisiográficas que presentan condiciones de aptitud ecológica para la guacamaya durante el periodo 2002-2008. Su distribución actual se analizó mediante sistemas de información geográfica y su distribución potencial se obtuvo mediante el algoritmo genético para producción de reglas (GARP). Se evaluó la coincidencia entre registros de campo y la distribución de condiciones ecológicas propicias (elevación, vegetación) para la especie, generadas mediante modelaje ecológico de nicho. Se confirmó su presencia en los municipios de La Huacana, Churumuco y Arteaga, su distribución en 2359 km² de la región conocida como bajo Balsas y se obtuvieron registros en la sierra Madre del Sur; sin embargo, todavía existen grandes extensiones que requieren verificación de campo, por lo que la distribución de la guacamaya para esta zona todavía está considerada como potencial. La guacamaya verde habitó en la costa michoacana hasta 1980, actualmente ha sido extirpada de un área que se estima en $1303 \mathrm{~km}^{2}$. Considerando la superficie de distribución confirmada y la superficie con aptitud que no ha sido verificada, la especie podría contar con $8285 \mathrm{~km}^{2}$ de distribución en el estado. Adicionalmente se localizaron 7 áreas de descanso y anidación de la especie. El área conocida como Chorros del Varal, en el municipio de Los Reyes colinda con el estado de Jalisco y podría ser un área de contacto entre poblaciones de guacamayas de los 2 estados. Este es el primer estudio estatal en el país que combina registros de campo actuales y modelos ecológicos de nicho para estimar la distribución real y potencial de la especie.

Palabras clave: Ara militaris, distribución confirmada y potencial, áreas de anidación, área de extirpación.

\begin{abstract}
The military macaw (Ara militaris) is an endangered species in Mexico, it has disappeared from states on the south Pacific. However, actual studies providing distribution information on the different states are inexistent. The main objective of this study was to provide information concerning its actual distribution on Michoacán. We evaluated 215 localities from 3 physiographic regions that present suitable ecological conditions during the 2002-2008 period. Its actual distribution was analyzed using geographic information systems, and its potential distribution was obtained with the genetic algorithm for rule set prediction (GARP). We evaluated the coincidence between field records and the distribution of suitable ecological conditions (elevation, vegetation) for the species, generated by ecological niche modeling. We confirmed its presence on the Municipalities of La Huacana, Churumuco, and Arteaga, confirming its distribution on $2359 \mathrm{~km}^{2}$ from the region known as the lower Balsas basin. In addition, we obtained records on the Sierra Madre del Sur, however there are large areas from this region that require field confirmation, and the distribution for this region can still be considered as potential. The military macaw inhabited the Michoacán coast in 1980, today the species its been extirpated from an estimated area of $1303 \mathrm{~km}^{2}$. Considering the extent of the confirmed distribution, and the extent of the area with suitable ecological conditions that require field confirmation, we assume that the species may have $8285 \mathrm{~km}^{2}$ of distribution on the state. Additionally we localized 7 roosting and nesting areas of the species. The area known as Chorros del Varal, in the municipality of Los Reyes is important because is on the border limits with Jalisco state and could be a contact area between macaw populations of the 2 states. This is the first state level study in the country that combines actual field records and ecological niche modeling to estimate the species actual and potential distribution.
\end{abstract}

Key words: Ara militaris, actual and potential distribution, nesting localities, extirpation area. 


\section{Introducción}

Ante la carencia de información actual de campo para la mayoría de especies en peligro de extinción, como es el caso de la guacamaya verde (Ara militaris), recientemente se han desarrollado un conjunto de metodologías en las que se estima la distribución potencial de las especies mediante el uso de modelos ecológicos de nicho. En estos modelos se evalúan cambios hipotéticos en la distribución de las especies asociados a cambios en sus hábitats dentro de áreas aptas para su presencia (Peterson et al., 2006; Ríos-Muñoz y Navarro-Sigüenza, 2009). En estas metodologías generalmente se utilizan datos de colecciones científicas de las especies de interés, capas digitales de las variables ambientales y algoritmos como GARP, en Sistemas de Información Geográfica que permiten generar hipótesis sobre la posible distribución de estas especies. Entre sus congéneres, la guacamaya verde (Ara militaris) es la especie que presenta la distribución más norteña en el continente americano. Aparece catalogada como vulnerable y en peligro de extinción en la lista internacional y en la nacional de especies amenazadas, respectivamente (Snyder et al., 2000; INE-SEMARNAP, 2000). En México, su distribución histórica se daba en el Pacífico, desde el sur de Sonora a lo largo de la sierra Madre Occidental y sierra Madre del Sur, incluyendo algunas regiones costeras en tierras bajas, hasta los estados de Oaxaca y Chiapas, donde se estima que fue extirpada a inicios del siglo pasado (Álvarez del Toro, 1952; Gardner, 1972; Binford, 1989). Su distribución en el golfo de México incluyó la sierra Madre Oriental desde el este de Nuevo León hasta el noreste de Querétaro (Forshaw 1989; Howell y Webb, 1995; Russell y Monson, 1998). En el país habita en bosques tropicales deciduos, subdeciduos y subperenifolios, desde el nivel del mar hasta los $1500 \mathrm{~m}$ y en áreas templadas de pinoencino hasta los $2200 \mathrm{~m}$. También habita en ambientes semiáridos de la depresión del Balsas, centro de Sonora y la región de Tehuacán-Cuicatlán en Oaxaca. Anida en cavidades de árboles emergentes y acantilados (Baker, 1958; Binford, 1989; Carreón-Arroyo, 1997; De LabraHernández, 2007; Rivera-Ortíz et al., 2008).

En México, el potencial de estas metodologías se ilustró con 2 estudios recientes a escalas geográficas muy amplias con aves de las familias Corvidae y Psittacidae, en los que se analizan los cambios de la cobertura vegetal en diferentes periodos y su coincidencia con la distribución hipotética de las especies generada con modelos ecológicos de nicho (Peterson et al., 2006; Ríos-Muñoz y Navarro-Sigüenza, 2009). Estos estudios plantean hipótesis de cambios en la distribución asociados a cambios en la cobertura del hábitat primario de las especies. Se estimó que 8 de 11 especies de Corvidae analizadas han perdido hasta un 35\%.de hábitat en sus distribuciones potenciales en los casos más drásticos Un resultado similar se estimó para la familia Psittacidae en Mexico, con pérdida de hábitat para 19 de 22 especies analizadas, hasta del $85 \%$ en los casos más drásticos. Sin embargo, el método presenta algunas limitaciones, al realizarse a gran escala se pierden detalles locales y se puede sobrestimar o subestimar la distribución de las especies. Por ejemplo, en el caso de los Psitácidos, una especie puede ser extirpada de una región aún en presencia de hábitat primario. Este caso se documentó para el loro cabeza amarilla, Amazona oratrix, en la costa de Michoacán, donde se estimó que la tercera parte de pérdida de distribución de la especie no se debió a la reducción del hábitat y cambios de uso de suelo, sino al fuerte comercio ilegal y saqueo de nidos, ya que áreas donde fue extirpada todavía presentaban hábitat para su presencia (Monterrubio-Rico et al., 2007).

En el caso de Ara militaris, su pérdida de distribución en la costa del Pacífico de México se ha dado desde principios de la década de 1980, registrándose declive y desaparición de sus poblaciones sin que existieran estudios específicos que proporcionaran más detalles (Ridgely, 1981). Además de la pérdida de distribución confirmada en Oaxaca y Chiapas, se estima que la especie ha sido extirpada de extensas áreas de zonas costeras de Sinaloa, Nayarit, Jalisco y Michoacán en los últimos 30 años (Ridgely, 1981; Villaseñor-Gómez, 1988; Navarro-Sigüenza, 1998; INE-SEMARNAP, 2000). El estudio realizado para todo el país por Ríos-Muñoz y Navarro-Sigüenza (2009), basado en modelos ecológicos de nicho y cambios de uso de suelo, estimó que la guacamaya verde ha perdido el $29 \%$ de su hábitat y que presenta distribución potencial en $160000 \mathrm{~km}^{2}$. Sin embargo, no existen todavía estudios de campo que confirmen las predicciones para los estados y se desconoce aún su distribución en cada estado y región del país.

En el caso de Michoacán, se desconoce la distribución de la guacamaya verde, existe una preocupación particular por la pérdida de distribución en Guerrero, Oaxaca y Chiapas y por la elevada deforestación experimentada en la costa del Pacifico mexicano (Ridgely, 1981; NavarroSigüenza, 1998; Trejo y Dirzo, 2000).

Ante la vulnerabilidad de la especie, su conservación requiere de información actual y precisa sobre su distribución, por lo que se ha actualizado la información para el estado de Michoacán, combinando en un enfoque metodológico modelos ecológicos de nicho y registros actuales de campo. Por lo inaccesible y peligroso de ciertas regiones, se indican las áreas que presentan capacidad ecológica para la distribución potencial de la especie y que requieren de verificación en campo. 
En este estudio se proporcionan estimaciones de distribución confirmada de la guacamaya verde y se indican las áreas donde la especie ha sido extirpada, así como los sitios de descanso y anidación. En México existen pocos estudios de los estados donde los resultados de modelos ecológicos de nicho sean validados con registros de campo, por lo que aquí se ilustran los beneficios de combinar trabajo de campo y modelos de distribución geográfica.

\section{Materiales y métodos}

Se consultaron bases de datos del Laboratorio de Investigación en Ornitología de la Facultad de Biología de la Universidad Michoacana de San Nicolás de Hidalgo y se evaluaron las regiones que presentaban registros históricos y condiciones para la presencia potencial de la especie, como elevación, topografía y vegetación (Howell y Webb, 1995). Se realizaron recorridos exploratorios y conteos durante el periodo 2001-2008 en 3 regiones fisiográficas del estado de Michoacán (Fig. 1). Los métodos en campo incluyeron conteos matutinos mediante una técnica mixta de puntos de conteo y transectos, se establecieron rutas de $2.4 \mathrm{~km}$, a lo largo de las cuales se realizaron 12 puntos de conteo, con distancia de $200 \mathrm{~m}$ entre puntos. También se anotaron las observaciones generadas durante el trayecto entre puntos. Los conteos se realizaron de 7:00. a 10:30, horas de actividad en las especies de psitácidos. Cada punto de conteo duró 10 minutos; se registró número de individuos, hora de avistamiento, distancia y orientación del observador al ave detectada, actividad (percha, forrajeo o vuelo) y observaciones adicionales, como las especies de árboles que utilizaron para perchar o alimentarse y se de registraron las coordenadas geográficas y la altitud del inicio y final del transecto (Bibby et al., 2000).

También se efectuaron conteos vespertinos desde puntos fijos elevados, desde sitios con un ángulo de visión entre $180^{\circ}$ y $360^{\circ}$ y por encima del dosel de la vegetación; las parvadas que se dirigían a los sitios de alimentación vespertina, descanso o anidación se observaron con mayor precisión. En cada detección se registraron las mismas variables que en los puntos de conteo. Los conteos se realizaron de las 17:30 a las 19:30 horas (Bibby et al., 2000). Por último, se realizó búsqueda intensiva de sitios de anidación con el apoyo de habitantes de la región, quienes ocasionalmente capturan pollos para su comercialización. Se buscó conducta reproductiva, como la actividad intensa de parejas en torno a cavidades de los acantilados, inspección y permanencia por más de 20 minutos en cavidades, vuelo de tríos en la etapa de reclutamiento, distinguiéndose el vuelo errático de las supuestas crías (Rivera-Ortíz et al., 2008). Se documentó también la presencia de pollos en caseríos cercanos.

Las regiones estudiadas fueron: las tierras bajas $(\leq$ $500 \mathrm{~m}$ ) de la costa del Pacífico (Municipios de Coahuayana, Aquila y Lázaro Cárdenas), la cuenca baja del río Balsas (Churumuco, La Huacana, Arteaga), los municipios de Periban y Los Reyes alrededor de los $1000 \mathrm{~m}$ snm, en las inmediaciones del Tancitaro y diversas localidades

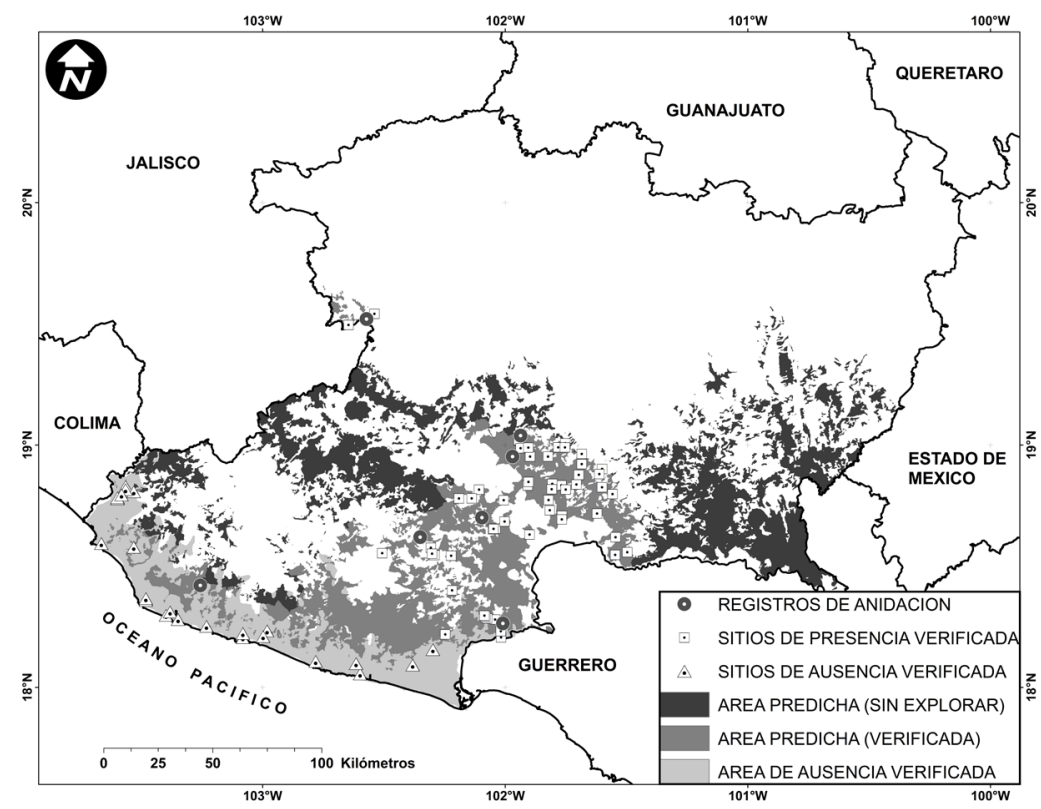

Figura 1. Distribución de la guacamaya verde en Michoacán, se indican áreas de distribución confirmada, distribución potencial y áreas de extirpación. 
de la sierra Madre del Sur (Chinicuila, suroeste de Tumbiscatío, suroeste de Arteaga y parte alta de Aquila). El esfuerzo de campo fue similar entre la época reproductiva (marzo-junio) y la no reproductiva (agosto-febrero), con 50 días por temporada para la región de la costa (20022007) y 90 días por temporada para la región del bajo Balsas (2006-2008). Para la región de la sierra Madre del Sur $(\geq 1000 \mathrm{~m})$, se realizó un esfuerzo de campo de 30 días durante 2007-2008. Debido a los altos niveles de inseguridad registrados en Aguililla, Coalcomán, Tepalcatepec y Apatzingán, las áreas con cobertura de bosques tropicales conservados de estos municipios no fueron visitadas en campo. Sin embargo, se exploraron las tierras bajas $(\leq 1000 \mathrm{~m})$ de la vertiente del Pacífico de la sierra Madre del Sur desde la costa en Aquila y desde el bajo Balsas en Arteaga. Las localidades visitadas se seleccionaron de acuerdo con criterios ecológicos (vegetación y elevación adecuadas), de accesibilidad (caminos rurales, brechas, ríos) y de seguridad.

Modelos predictivos. Se utilizó un modelo de nicho ecológico (ENM) para generar una estimación sobre la distribución potencial de la especie, con base en la proyección de las condiciones de aptitud ecológica asociadas a la distribución observada de la especie en los municipios accesibles y prediciendo en el interior de municipios inaccesibles. El modelado de nicho ecológico se considera actualmente el enfoque más completo para predecir distribuciones potenciales de las especies, condiciones de aptitud ecológica para las especies, o disponibilidad del hábitat adecuado (Guisan y Zimmermann, 2000; Peterson, 2001; Guisan y Thuiller, 2005; Soberón y Peterson, 2005). Para una especie como la guacamaya verde, asociada a vegetación donde anida y se alimenta, el empleo de registros recientes de áreas de hábitat remanente y modelar su distribución se considera el mejor enfoque para estimar su distribución actual (Chen y Peterson, 2000).

Modelado de nicho ecológico de la guacamaya verde. Se seleccionó GARP entre los algoritmos existentes, porque bajo condiciones difíciles es preciso, presentando un buen desempeño en estudios comparativos (Papes y Gaubert, 2007; Peterson et al., 2007; Tsoar et al., 2007); se utilizaron los procedimientos estándar de la metodología GARP (Stockwell y Peterson, 2002; Anderson et al., 2003). En los modelos se emplearon 7 capas ambientales (proyecciones geográficas y datum WGS84), incluyendo 4 obtenidas de la base de datos Hydrolk (http://edc.usgs.gov/products/ elevation/gtopo30/hydro/index.html): elevación, aspecto, pendiente y un compuesto de índice topográfico;además, 3 capas de campo continuo de vegetación VCF (http://glcf. umiacs.umd.edu/data/vcf/index.shtml), el cual contiene estimaciones proporcionales de los tipos de cobertura vegetal: vegetación arbolada, herbácea y suelo desnudo, todos derivados de imágenes de satélite MODIS. Una vez construidos los modelos, se evaluaron los tipos de vegetación utilizando la información digital del Inventario Forestal Nacional 2000 y la información de campo (Velásquez et al., 2002). Las capas de datos se ajustaron a 30" de tamaño de celda $(\approx 1 \mathrm{~km})$. Para generar los modelos se utilizó el $50 \%$ de los registros de campo, de los cuales internamente GARP utilizó el $60 \%$ de los puntos para training y el $40 \%$ para testing. Se generaron 100 modelos, seleccionándose los 10 con el menor error de omisión $(<0.05 \%$ de omisión intrínseca). Con el auxilio de sistemas de información geográfica, los 10 modelos seleccionados se sumaron para obtener un mapa compuesto (mapa de congruencias o aptitudes), en el cual el valor de cada celda (pixel) representa el número de modelos independientes que proyectaron presencia de la especie para la celda (Anderson et al., 2003). Para la validación externa de los modelos se sobrepuso el $50 \%$ de los registros de presencia; un modelo utilizable es el que anticipa la distribución del conjunto de registros independientes (Peterson, 2001). Cuando se trata de una especie en peligro de extinción, es recomendable que los modelos tengan el menor nivel de error de comisión, es decir, que no sobreestimen la distribución y utilicen un umbral conservador respecto a las áreas de presencia proyectada (Papes y Gaubert, 2007). Se estableció un umbral analizando la coincidencia de los registros de campo con el número de modelos independientes que proyectaron presencia de la especie en nivel de pixel. En los modelos más precisos el $97 \%$ de los registros de campo coincidieron con 8-10 modelos que proyectaron presencia potencial. Por lo que se considera como el área de mayor presencia potencial a la superficie (píxeles) donde coinciden de 8 a 10 modelos.

Para cuantificar los tipos de vegetación y usos de suelo presentes en las áreas de aptitud que proyectaron los modelos y posteriormente analizarlos en base a su disponibilidad como hábitat potencial para la guacamaya verde, se utilizaron las estadísticas de vegetación y uso de suelo del Inventario Forestal Nacional (Velázquez et al., 2002). En este estudio se consideran 4 categorías: bosques templados, tanto conservados como perturbados (combinaciones de bosques de coníferas y encinares), tierras agropecuarias (todos los tipos de agricultura, ganadería y huertas), bosque tropical caducifolio (conservado y perturbado) y bosque tropical subcaducifolio y subperenifolio, incluyendo aquellos que aparecen en el inventario como perturbados.

\section{Resultados}

Distribución potencial actual. Se visitó un total de 215 localidades (coordenadas únicas) en 3 regiones del estado. La presencia de la especie se confirmó en 65 localidades 
(30\%) de las regiones del bajo Balsas y sierra Madre del Sur. En la del bajo Balsas, se observó en 58 de las 94 (59.2\%) localidades, de los municipios de La Huacana, Churumuco y Arteaga y que constituyen su distribución confirmada en campo. Para la región de la Costa no se obtuvo ningún registro de la especie en 82 localidades visitadas. Respecto a la sierra Madre del Sur, se confirmó que la especie está presente en el municipio de Tumbiscatío; se le observó en 6 de 35 localidades evaluadas (Cuadro 1). Las observaciones de las guacamayas ocurrieron entre los 160 y los $1500 \mathrm{~m}$, con el $44 \%$ de los registros entre los 160 y los $400 \mathrm{~m}$ y el $20.6 \%$ entre los 880 y $1500 \mathrm{~m}$. En la región del bajo Balsas se le observó entre los $160 \mathrm{~m}$ y 1 $268 \mathrm{~m}$; en el municipio de los Reyes, al límite con Jalisco, entre los $890 \mathrm{~m}$ y $1000 \mathrm{~m}$ y en la sierra Madre del Sur entre los $890 \mathrm{~m}$ y los $1500 \mathrm{~m}$.

El modelo ecológico de nicho generó una predicción de condiciones ecológicas aptas para la presencia potencial de la especie en el estado en $9588 \mathrm{~km}^{2}$. La región para la que se proyectó una mayor extensión fue el bajo Balsas con $5485 \mathrm{~km}^{2}(57 \%$ de la superficie en el estado con aptitud ecológica), seguido de la sierra Madre del Sur con 2800 $\mathrm{km}^{2}$ y la región de la Costa con $1303 \mathrm{~km}^{2}$. Sin embargo, en el muestreo de campo se confirmó que la especie ha sido extirpada de la región de la Costa Michoacana, de zonas a baja elevación $(\leq 800 \mathrm{~m})$ de los 3 municipios (Coahuayana, Aquila y Lázaro Cárdenas). Por lo que la superficie que los modelos ilustran con aptitud ecológica para la especie se consideró como superficie de pérdida de distribución, ya que existe evidencia de su presencia hace 30 años (Villaseñor, 1988). En el municipio de Aquila se obtuvo un registro, pero a una elevación de $890 \mathrm{~m}$, por lo que fisiográficamente corresponde a la sierra Madre del Sur (Fig. 1). En la superficie con aptitud proyectada en el bajo Balsas, sólo se consideraron como superficie confirmada 2359 $\mathrm{km}^{2}$, que corresponden al área de coincidencia de registros de campo para los municipios de Huacana, Churumuco y Arteaga. Esta superficie representa el $43 \%$ de la predicción para esta región, quedando pendiente de verificación una superficie de $3126 \mathrm{~km}^{2}$ en los municipios de Huetamo, San Lucas, Carácuaro, Nocupétaro, Tiquicheo, Turicato y el sur de Tacámbaro. En la sierra Madre del Sur el esfuerzo de campo es todavía poco representativo. La ubicación de las localidades no cubrieron la totalidad del municipio de Tumbiscatio, por lo que no se considera como distribución confirmada el área que presenta condiciones de aptitud con base en el modelo. En esta región quedan pendientes de verificación de campo los municipios de Aguililla, Coalcomán, Tepalcatepec y suroeste de Apatzingán. Eliminando la distribución potencial proyectada para la Costa, la especie presenta una distribución confirmada de $2359 \mathrm{~km}^{2} \mathrm{y}$ una distribución potencial adicional de $5926 \mathrm{~km}^{2}$.
Distribución observada y potencial por tipo de vegetación. El mayor porcentaje de observaciones de la especie se obtuvo en bosque tropical caducifolio $(n=36)$; representan el 55.3\% del total de registros, con 33 registros de la región del Bajo Balsas y 3 de Tumbiscatio (Sierra Madre del Sur). Estos últimos ocurrieron en ecotonos con presencia de bosque templado de pino-encino. Los bosques templados fueron el segundo tipo de vegetación natural, donde las guacamayas se observaron desde los $890 \mathrm{~m}$ en ecotonos con bosque tropical caducifolio, hasta bosques de pino-encino a los $1500 \mathrm{~m}$ (Cuadro 1); 3 se registraron en la Sierra Madre del Sur y 3 en la región del Bajo Balsas. Sólo 4 de los registros se obtuvieron en bosque tropical subperenifolio-subcaducifolio, en el extremo noroeste de la depresión del Balsas, municipios de Los Reyes y Periban, al límite con el eje neovolcánico transversal. En superficie agropecuaria se obtuvieron 19 registros (Cuadro 1). En las áreas de aptitud ecológica que proyectaron los modelos para la presencia potencial de la especie, los tipos de vegetación fueron: bosque tropical caducifolio (49.3\%), superficie agropecuaria (34\%), bosque tropical subperenifolio-subcaducifolio (11\%) y bosques templados (4.6\%).

Además de los registros, se localizaron 7 localidades de descanso y anidación de la guacamaya verde, 5 en cavidades de acantilados de montañas escarpadas en los municipios de La Huacana (2) y Arteaga (1) en el Bajo Balsas, 2 en la sierra Madre del Sur, en Aquila (1) y Tumbiscatío (1) y en 2 localidades en paredes de cañadas de los municipios de Los Reyes y La Huacana, respectivamente (Fig.1). Las zonas fueron visitadas durante 3 años al inicio de la época reproductiva (febrero-marzo). Los tipos de vegetación en 2 sitios de anidación de La Huacana correspondieron a bosque de encino-pino y bosque tropical subperenifolio respectivamente. En las localidades de Aquila y Tumbiscatío las cavidades se encontraron en un ecotono de bosque tropical caducifolio y bosque de encino-pino. El sitio de anidación del municipio de Los Reyes, es una cañada (820 a 1160 m snm), en cuyo fondo

Cuadro 1. Registros de campo por tipo de vegetación y porcentajes de cobertura potencial de cada categoría de vegetación en el modelo ecológico de nicho de guacamaya verde

\begin{tabular}{lcc}
\hline Tipos de vegetación & $\begin{array}{c}\text { Núm. registros } \\
\text { (\%) }\end{array}$ & $\begin{array}{c}\text { Porcentaje } \\
\text { en modelo }\end{array}$ \\
\hline $\begin{array}{l}\text { Bosque tropical } \\
\text { caducifolio }\end{array}$ & $36(55.3)$ & 49.3 \\
$\begin{array}{l}\text { Bosque tropical } \\
\text { subperenifolio- }\end{array}$ & $4(6.1)$ & 11 \\
$\begin{array}{l}\text { subcaducifolio } \\
\begin{array}{l}\text { Bosques templados } \\
\text { Superficie agropecuaria }\end{array}\end{array}$ & $6(9.2)$ & 4.6 \\
\hline
\end{tabular}


convergen los ríos Apupataro e Itzicuaro. La cañada presenta cavidades de alrededor de $70 \mathrm{~m}$ respecto al fondo, donde predomina el bosque tropical subperenifolio. En el tercer sitio de anidación de La Huacana, que corresponde a una cañada, se encontró una mezcla de bosque tropical caducifolio y bosque tropical subperenifolio.

\section{Discusión}

En este estudio se confirma la presencia de la especie en 2 regiones de Michoacán, distribuyéndose de manera potencial sobre una superficie relativamente extensa en 2 de las 3 regiones de su presencia histórica. Sin embargo, la distribución confirmada sólo equivale al 29\% de la superficie que los modelos indican con condiciones aptas para la especie. Todavía se requiere verificación de campo sobre extensas áreas de la región del bajo Balsas y la sierra Madre del Sur. Si se confirmara su presencia en la totalidad del área faltante de distribución potencial no verificada, la especie podría presentar una extensión de $8286 \mathrm{~km}^{2}$ que es el 28.4\% del área de las 3 regiones incluidas en el análisis.

A pesar de sus limitaciones, éste es el primer estudio de escala estatal en México que proporciona cifras actualizadas sobre la distribución de la especie, e identifica la contracción en su distribución de manera precisa. En la región de la costa se desarrolló un esfuerzo extensivo de muestreo y los resultados revelaron que la especie ha desaparecido de esa región. Se plantea la hipótesis de que la especie ha sido extirpada de una extensión equivalente a la que los modelos proyectan con aptitud ecológica y que representa el $13.5 \%$ del área potencial total para la especie en el estado.

Es importante destacar que en la costa la guacamaya ha sido extirpada en presencia de extensas áreas de bosque tropical caducifolio y bosque tropical subperenifolio, tipos de vegetación en que se generaron registros en las otras 2 regiones de estudio y que son significativas para la conservación de la especie en otras partes del país (Carreón-Arroyo, 1997; Rivera-Ortiz et al., 2008), por lo que su pérdida en la costa Michoacana no se puede atribuir únicamente a pérdida de hábitat. Adicionalmente, puede atribuirse a otras causas como el saqueo para el mercado de mascotas, la caza y la introducción de depredadores y enfermedades (Snyder et al., 2000). Los habitantes de mayor edad en la región refieren haber visto parvadas de guacamayas verdes en los municipios de Aquila y Lázaro Cárdenas, todavía durante los años de 1970.

El registro de la localidad llamada Chorros del Varal, en el municipio de Los Reyes, es particularmente importante por su ubicación. Además de colindar con Jalisco, es una zona donde también convergen montañas y cañadas que se conectan en distintas direcciones y regiones. Aunque fisio- gráficamente corresponde al extremo más noroccidental de la depresión del Balsas, también es una zona donde colinda la depresión del Tepalcatepec y el Eje Neovolcánico Transversal. La presencia de guacamayas en las barrancas de esta localidad permiten plantear la hipótesis de una posible conectividad entre poblaciones de Michoacán y Jalisco que utilizan las cañadas para desplazarse. Esta hipótesis debe verificarse, ya que la especie está perdiendo distribución en el sur, por el Pacífico y es importante identificar áreas de contacto entre poblaciones de estados vecinos para establecer una estrategia de conservación.

Los diagnósticos estatales sobre especies en peligro de extinción, como el aquí presentado, complementan los análisis del país, ya que proporcionan mayor detalle y permiten confirmar o corregir las hipótesis de distribución generadas a mayor escala, aportando datos locales y municipales. El modelo predictivo generado en este estudio es preciso, ya que los registros de campo empleados en validación externa al modelo coincidieron con las áreas geográficas de aptitud ecológica proyectadas. Por esta razón, las áreas con predicción de aptitud ecológica para la especie, pueden considerarse como áreas para su distribución potencial. Ante la carencia de datos, este criterio es importante, ya que permite evaluar regiones inaccesibles. Considerando como distribución actual sólo aquellas áreas donde en campo se verificó la presencia de la especie, la utilización de un enfoque que integra datos de campo recientes y modelos predictivos permitió tener un diagnóstico para todo el estado que incluye áreas inaccesibles y peligrosas que de otra forma no se hubieran podido evaluar. Este enfoque también permitió identificar 3 tipos de áreas asociadas a la distribución de la especie: 1), de distribución actual confirmada; 2), de extirpación de la especie y 3), de distribución potencial que requieren verificación de campo. La identificación de estas áreas permite visualizar acciones concretas de investigación y conservación para cada una de ellas. La estimación de distribución y su modelo asociado pueden ser más precisos a medida que se disponga de más información proveniente de los municipios que no han sido verificados en campo.

La distribución de la guacamaya verde observada en el estado coincide poco con áreas importantes para la conservación de Amazona oratrix y Amazona finschi. En la localidad de Los Reyes la guacamaya comparte la barranca donde anida con el loro Amazona finschi y en algunas partes de La Huacana e Infiernillo coincide en barrancas y sitios de registro de Amazona oratrix. Sin embargo, las 2 especies de loro presentan sus áreas de anidación y poblaciones más importantes en la región de la costa y en la sierra Madre del Sur en el municipio de Arteaga (Monterrubio-Rico et al., 2007; Monterrubio-Rico et al., 2009; Alvares-Jara, 2010), mientras que la mayoría de los 
registros y sitios de anidación de la guacamaya verde se presentaron en el bajo Balsas y en la sierra Madre del Sur en Tumbiscatio. Para la guacamaya verde se requiere conservar áreas que coinciden poco con áreas de anidamiento de loros Amazona.

Por las diferencias de muestreo entre regiones y tipos de vegetación, no es posible realizar análisis estadísticos de uso y disponibilidad entre los tipos de vegetación disponibles, los utilizados y los porcentajes de vegetación en que se proyecta aptitud para distribución potencial de la guacamaya en los modelos. Sin embargo, existieron importantes coincidencias y las diferencias existentes son pequeñas. Los registros de campo y la vegetación con mayor extensión en los modelos predictivos coincidieron en el bosque tropical caducifolio como el principal tipo de vegetación. Aunque la superficie agrícola ocupa una extensión importante en las áreas de distribución de la guacamaya (segunda categoría en los modelos), en este estudio no se obtuvo evidencia de que sean utilizados como hábitat, los registros fueron relativamente pocos y no se le observó alimentándose en cultivos o huertos. Tampoco se le observó perchar en árboles de pastizales ganaderos. A las guacamayas se les observó volar sobre matrices de paisaje fragmentado con presencia de vegetación nativa y superficie agrícola. En contraste, aunque los bosques templados en los modelos ocuparon un porcentaje relativamente bajo de la distribución potencial de la especie, en estos y en ecotonos con bosque tropical caducifolio, se obtuvo un porcentaje de registros mayor que el porcentaje en que se proyectó en los modelos. La sierra Madre del Sur cuenta con amplias zonas de contacto entre bosques templados y bosques tropicales, ya que colinda con las regiones costa y depresión del Balsas, a medida que se verifique a la especie en esta región, se podrá evaluar mejor el valor de importancia de los bosques templados para la especie. El bosque tropical subperenifolio-subcaducifolio presentó en los modelos un porcentaje mayor que el porcentaje de registros de campo. En presente estudio se obtuvieron pocos registros de la especie en este tipo de vegetación; sin embargo, es la vegetación que predomina en el área de anidación de Chorros del Varal en Los Reyes y es donde se le observó alimentándose. Las diferencias pueden atribuirse a que estos bosques se localizan principalmente en la región de la costa, que es la región donde la especie ha sido extirpada. El bosque tropical subperenifolio es un hábitat importante para la conservación de Amazona finschi y Amazona oratrix por la disponibilidad de árboles nido y también se ha registrado como importante para la guacamaya verde en otros estados del país, como en Jalisco (Carreón-Arroyo, 1997). Es posible que si de manera efectiva se conservan áreas para las 2 especies de loros en la región de la costa, también se beneficie en el mediano y largo plazo la guacamaya verde, mediante una potencial reintroducción y si se identifican y corrigen las causas que originaron su desaparición. Existen experiencias exitosas de reintroducción de guacamayas escarlata (Ara macao) en tipos de vegetación de Costa Rica que por estacionalidad y estructura son similares a los de la costa michoacana (Myers y Vaughan, 2004; Brightsmith et al., 2005). En políticas gubernamentales, la región de la costa está destinada a ser un polo de desarrollo en ecoturismo, por lo que el establecimiento de poblaciones de guacamayas en la región constituiría un atractivo que podría contar con un amplio apoyo de las comunidades.

El diagnóstico del estadoes importante para el país, ya que de agravarse las tendencias de desaparición de la especie en Guerrero, Michoacán podría constituirse en el área con distribución confirmada de mayor extensión al sur, en el Pacífico, y por su ubicación podría ser estratégica para la conservación de la especie en todo el occidente (Ridgely, 1981; Navarro-Sigüenza, 1998).

En Michoacán, la investigación sobre esta especie debe completar el diagnóstico sobre su distribución actual en la sierra Madre del Sur, e inventariar sitios de anidación en el estado. Adicionalmente, debe conocerse la relación entre disponibilidad de hábitat y alimento con la abundancia de la especie. Es recomendable generar nuevos modelos de distribución a medida que se disponga de información proveniente de las regiones y municipios donde se predicen condiciones ecológicas aptas para la especie. Por último, se debe analizar la viabilidad de programas de reproducción en semicautiverio, para su posterior reintroducción en la región de la costa Michoacana, ya que con base en la disponibilidad de hábitat, sería una región idónea.

\section{Agradecimientos}

Este proyecto no hubiese sido posible sin la asistencia y colaboración de un amplio número de personas, particularmente Edna López Córdova, Beneiza Fabián Turja, Berenice Santiago Valencia, Lorena Téllez García, Alejandra Huerta Heredia, Karla Patricia Parra Noguez, Ismael Carrillo Acevedo, Margarito Álvarez Jara, Oliverio Delgado Carrillo, Guillermo Alberto Sánchez Rivera, Salvador Amezcua Ceja y Mario Suárez Suárez. Se contó con apoyo financiero del Fondo Sectorial de Investigación Ambiental CONACYT-SEMARNAT, otorgado al proyecto "Ecología y situación actual de las especies prioritarias de psitácidos de la vertiente del Pacífico mexicano", CONACYT-SEMARNAT-2002-C0021. Agradecemos a la Facultad de Biología de la Universidad Michoacana de San Nicolás de Hidalgo el continuo apoyo y a la Dirección General de Vida Silvestre por proporcionar los permisos de investigación. 


\section{Literatura citada}

Alvares-Jara, M. 2010. Ecología y distribución potencial de la familia Psittacidae en una zona de transición templadotropical en el bajo Balsas, Michoacán. Tesis, Facultad de Biología, Universidad Michoacana de San Nicolás de Hidalgo. Morelia, Michoacán. 54 p.

Álvarez del Toro, M. 1952. New records of birds from Chiapas, Mexico. Condor 54:112-114.

Anderson, R. P., D. Lew y A. T. Peterson. 2003. Evaluating predictive models of species distributions: criteria for selecting optimal models. Ecological Modeling 162:211-232.

Baker, R. H. 1958. Nest of the military macaw in Durango. Auk 75:98.

Bibby, C. J., N. D. Burgess, D. A. Hill y S. H. Mustoe. 2000. Bird census techniques, segunda edición.. Academic, London. $302 \mathrm{p}$.

Binford, L. 1989. A distribution survey of the birds of the state of Oaxaca. Ornithological Monographs 43. The American Ornithologists' Union, Washington, D. C. 418 p.

Brighttsmith, D., J. Hilburn, A. del Campo, J. Boyd, M. Frisius, R. Frisius, D. Janik y F. Guillen. 2005. The use of hand raised psittacines for reintroduction: a case study of scarlet macaws (Ara macao) in Peru and Costa Rica. Biological Conservation 121:465-472.

Carreón-Arroyo, G. 1997. Estimación poblacional, biología reproductiva y ecología de la nidificación de la guacamaya verde (Ara militaris) en una selva estacional del oeste de Jalisco, México. Tesis, Facultad de Ciencias, Universidad Nacional Autónoma de México, México, D. F. p. 67.

Chen, G. J. y A. T. Peterson. 2000. A new technique for predicting distribution of terrestrial vertebrates using inferential modeling. Zoological research 21:231-237.

De Labra-Hernández, M. A. 2007. Distribución potencial de la guacamaya verde (Ara militaris), en el municipio de La Huacana, Michoacán. Tesis, Facultad de Biología, Universidad Michoacana de San Nicolás de Hidalgo, Morelia, Michoacán. 49 p.

Forshaw, J. M. 1989. Parrots of the world, tercera edición. Silvio Mattachhione, Ontario. 616 p.

Gardner, A. L. 1972. The occurrence of Streptoprocne zonaris albicincta and Ara militaris in Chiapas, México. Condor 74:480-481.

Guisan, A. y W. Thuiller. 2005. Predicting species distributions: offering more than simple habitat models. Ecology Letters 8:993-1009.

Guissan, A. y N. E. Zimmermann. 2000. Predictive habitat distribution models in ecology. Ecological Modeling 135:147-186.

Hansen, M., R. DeFries, J. R. Townshend, M. Carroll, C. Dimiceli y R. Sohlberg. 2006. Vegetation Continuous Fields MOD44B, 2001 Percent Tree Cover, Collection 4, University of Maryland, College Park, Maryland.

Howell, S. N. G. y S. Webb. 1995. A guide to the birds of Mexico and northern Central America. Oxford University Press, New York. 851 p.

INE-SEMARNAP (Instituto Nacional de Ecología-Secretaría de Medio Ambiente, Recursos Naturales y de Pesca). 2000. Proyecto para la conservación, manejo y aprovechamiento sustentable de los psitácidos en México. México, D. F. 145 p.

Monterrubio-Rico, T. C., J. M. Ortega-Rodríguez, M. C. MarínTogo, A. Salinas-Melgoza y K. Renton. 2009. Nesting hábitat of the Lilac-crowned Parrot in a modified Landscape. Biotropica 41:361-368.

Monterrubio-Rico, T. C., L. E. Villaseñor-Gómez, M. C. Marín-Togo, E. A. López-Cordova, B. Fabián-Turja y V. Sorani-Dalbón. 2007. Distribución histórica y actual del loro cabeza amarilla (Amazona oratrix) en la costa central del Pacífico mexicano: ventajas y limitaciones en el uso de GARP en especies bajo fuerte presión de tráfico. Ornitología Neotropical 18:263-276.

Myers, M. C. y C. Vaughan 2004. Movement and behavior of scarlet macaws (Ara macao) during the post-fledging dependence period: implications for in situ versus ex situ management. Biological Conservation 118:411-420.

Navarro-Sigüenza, A. G. 1998. Distribución geográfica y ecológica de la avifauna del estado de Guerrero, México. Tesis, doctorado Facultad de Ciencias, Universidad Nacional Autónoma de México, México, D. F. 182 p.

Papes, M. y P. Gaubert. 2007. Modelling ecological niches from low numbers of occurrences: assessment of the conservation status of poorly known viverrids (Mammalia: Carnivora) across two continents. Diversity and Distributions 13:890902.

Peterson, A. T. 2001. Predicting species geographic distributions based on ecological niche modeling. Condor 103: 599-605.

Peterson, A. T., V. Sánchez-Cordero, E. Martínez-Meyer y A. G. Navarro-Sigüenza. 2006. Tracking population extirpations via melding ecological niche modeling with land-cover information. Ecological Modelling 195:229-236.

Peterson, A. T., M. Papes y M. Eaton. 2007. Transferability and model evaluation in ecological niche modeling: a comparison of GARP and Maxent. Ecography 30:550-560.

Ridgely, R. S. 1981. The current distribution and status of mainland neotropical parrots. In Conservation of New World parrots. R. F. Pasquier (ed.) Smithsonian Institution Press/ ICBP, Washington, D. C. p. 233-384.

Ríos-Muñoz, C. A. y A. G. Navarro-Sigüenza. 2009. Efectos del cambio de uso de suelo en la disponibilidad de hábitat para los psitácidos de México. Ornitología Neotropical 20:491509.

Rivera-Ortiz, F. A., A. M. Contreras-González, C. A. SoberanesGonzález, A. Valiente-Banuet y M. del Coro-Arizmendi. 2008. Seasonal abundance and breeding chronology of the 
military macaw (Ara militaris) in a semiarid region of central Mexico. Ornitología Neotropical 19:255-263.

Russell, S. M. y G. Monson. 1998. The birds of Sonora. The University of Arizona Press. Tucson. 360 p.

Snyder, N. F. R., P. Mc Gowan, J. Gilardi y A. Grajal. 2000. Parrots. Status survey and conservation action plan 20002004. IUCN The World Conservation Union, Oxford, 180 p.

Soberón, J. y A. T. Peterson. 2005. Interpretation of fundamental ecological niches and species distributional areas. Biodiversity Informatics 2:1-10.

Stockwell, D. R. B. y T. A. Peterson. 2002. Effects of sample size on accuracy of species distribution models. Ecological Modelling 148:1-13.

Trejo, I. y R. Dirzo. 2000. Deforestation of seasonally dry tropical forest: a national and local analysis in Mexico. Biological Conservation 94:133-142.
Tsoar, A., O. Allouche, O. Steinitz, D. Rotem y R. Kadmon. 2007. A comparative evaluation of presence-only methods for modelling species distributions. Diversity and Distributions 13:397-405.

USGS. 2001. HYDRO1k Elevation Derivative Database, http:// eros.usgs.gov/\#/Find_Data/Products_and_Data_Available/ gtopo30/hydro. U.S. Geological Survey, Washington; última consulta: 03.XI.2009.

Velázquez, A., J. F. Mas, J. R. Díaz-Gallegos, R. MayorgaSaucedo, P. C. Alcántara, R. Castro, T. Fernández, G. Bocco, E. Ezcurra y J. L. Palacio. 2002. Patrones y tasas de cambio de uso de suelo en México. Gaceta Ecológica 62:21-37.

Villaseñor-Gómez, J. F. 1988. Aves costeras de Michoacán, México. Universidad Michoacana de San Nicolás de Hidalgo. Tesis Facultad de Biología,Universidad Michoacana de San Nicolás de Hidalgo, Morelia, Michoacán, México. 63 p. 